\title{
El Impacto del Uso de Pizarras Digitales Interactivas (PDI) en el Proceso de Enseñanza Aprendizaje. Un Caso de Estudio en la Universidad de Otavalo
}

\author{
Ramón Cala, Lidia I. Díaz*, Nora Espí y Jessica M. Tituaña \\ Universidad de Otavalo, Cdla. Imbaya y Av. de los Sarances $\mathrm{s} / \mathrm{n}$ y Pendoneros, Otavalo - Ecuador. \\ (e-mail: rcala@uotavalo.edu.ec; Idiaz@uotavalo.edu.ec; nespi@uotavalo.edu.ec; jtituania@uotavalo.edu.ec) \\ * Autor a quien debe ser dirigida la correspondencia
}

Recibido Nov. 29, 2017; Aceptado Feb. 12, 2018; Versión final Abr. 12, 2018, Publicado Oct. 2018

\begin{abstract}
Resumen
Es objetivo de este trabajo es reflexionar sobre la importancia del uso de las pizarras digitales interactivas (PDI), como recurso flexible y adaptable a diferentes estrategias docentes, que aportan al aprendizaje constructivista. Para corroborar sus efectos en el proceso de enseñanza aprendizaje en la Universidad de Otavalo, se realizó un estudio preliminar entre docentes y estudiantes, aplicándoles una encuesta a todos los profesores tiempo completo y a una muestra estratificada de estudiantes de la institución, con un nivel de confianza del $95 \%$. Se usó el paquete estadístico SPSS versión 21 para el procesamiento de la información, concluyéndose que tanto docentes como estudiantes consideran positivo el uso de las pizarras digitales interactivas en las aulas universitarias. No obstante, resulta imprescindible perfeccionar la metodología de integración de las PDI al proceso docente educativo en la Universidad de Otavalo, así como la planificación y realización de seminarios y talleres de socialización.
\end{abstract}

Palabras clave: pizarras digitales interactivas; proceso de enseñanza aprendizaje; estrategias docentes

\section{The Impact of the Use of Interactive Digital Screens (IDS) in the Teaching Learning Process. A Study Case in the Otavalo University}

\begin{abstract}
The purpose of this paper is to analyze and discuss about the importance of the use of interactive digital whiteboards (PDI), as a flexible and adaptable resource for different teaching strategies, which contribute to constructivist learning. To corroborate its effects on the teaching-learning process at the University of Otavalo, a preliminary study was conducted among teachers and students. A survey was applied to all full-time teachers and to a stratified sample of students of the institution, with a confidence level of $95 \%$ The statistical package SPSS version 21 was used for processing the collected information, concluding that both teachers and students consider positive the use of interactive whiteboards in university classrooms. However, it is essential to improve the methodology of integration of the PDI to the educational process in the University of Otavalo, as well as the planning and conducting of seminars and socialization workshops.
\end{abstract}

Keywords: interactive digital screens; teaching-learning process; teaching strategies 


\section{INTRODUCCIÓN}

Las tecnologías de la información y comunicación (TIC) ejercen un efecto revolucionario y transformador en la vida personal y profesional, en la medida que altera las formas de acceso al conocimiento y el aprendizaje. La sociedad de la información se ha convertido en factor de poder y productividad (Álvarez y Martinell, 2016). La productividad y la competitividad dependen cada vez más de la capacidad de generar y aplicar la información basada en el conocimiento. El mayor interés radica en la observación de la relación existente entre el uso de las TIC, fundamentalmente de Internet, y las modificaciones que se producen en las prácticas educativas, entendiendo en este caso, como sugieren De la Herran y Fortunato, (2017), que la clave no está en la tecnología ni tampoco en la pedagogía, sino en el uso pedagógico de la tecnología. Un aspecto fundamental resulta la aceptación por parte del usuario de los dispositivos TIC y el análisis de la relación beneficio- dificultades de su uso en el ámbito educativo, siendo superiores los beneficios, a criterio de los usuarios, que las dificultades que implican su implementación. (Yang, et al., 2016). Toda institución educativa sea de enseñanza media o superior, que tenga como imperativo central el perfeccionamiento de la calidad de su proceso de enseñanza aprendizaje, hará lo posible por incluir en sus aulas este recurso tecnológico, que brinda generalmente mayor posibilidad de aprendizaje al estudiante, (Murano, 2012).

Es difícil determinar todos los efectos que las tecnologías informáticas pueden tener sobre la educación, porque entre otras razones, no se ha desarrollado suficiente investigación para documentar estos efectos. Sobre la base de la revisión de la literatura, pueden identificarse al menos ocho tendencias básicas, que se expresan en los siguientes cambios: 1) de la instrucción global a la instrucción individualizada; 2) de la clase magistral y la exposición oral al entrenamiento y la instrucción; 3) de trabajar con los mejores alumnos a trabajar con los menos aventajados; 4) de estudiantes aburridos hacia estudiantes más comprometidos con la tarea; 5) de una evaluación basada en exámenes a una evaluación basada en productos, en el progreso y en el esfuerzo del alumno; 6) de una estructura competitiva a una cooperativa; 7) de programas educativos homogéneos a la selección personal de contenidos; 8) de la primacía del pensamiento verbal a la integración del pensamiento visual y verbal (Gallego et al., 2009),(Gómez y Aretio, 2016) (Basantes et al., 2017). La pizarra digital interactiva resulta el recurso tecno educativo, dentro de las TIC, que ha irrumpido con más fuerza en el entorno educacional y formación profesional en el siglo XXI. Sin embargo según Hernández, (Hernández, et al., 2014) "es importante reseñar que la incorporación de innovaciones tecnológicas por sí mismas no conduce a un mejoramiento de las prácticas educativas". Con mucha frecuencia se asocia el uso de las pizarras digitales interactivas (PDI) con una mayor motivación, compromiso y logro por parte de los estudiantes, pero muchos estudios ignoran el papel de profesor en efectuar esos cambios positivos (Stroud, Drayton, Hobbs y Falk, 2014). Si se quiere desarrollar todo su potencial, es necesario formular o reformular planteamientos pedagógicos adecuados con criterios de rentabilidad y eficacia didácticas (Gómez y Aretio, 2016).

Por tanto el problema a investigar está acotado al análisis de los criterios de docentes y estudiantes como elementos activos, respecto al uso de las PDI como recurso tecno pedagógico y su contribución al perfeccionamiento del proceso de enseñanza aprendizaje, tomando como caso de estudio la Universidad de Otavalo, Ecuador. Siendo las variables del estudio criterios de docentes y estudiantes sobre la significancia del uso de las PDI en el proceso de enseñanza aprendizaje en la Universidad de Otavalo, Ecuador.

\section{ANTECEDENTES}

La educación desde tiempos antiguos ha sufrido un sin número de adaptaciones a las exigencias sociales de cada época, por ende, en el siglo XXI el reto para este contexto es la incorporación positiva de nuevos recursos, contando ahora con las tecnologías de la información y comunicación (TIC) existiendo algunos de gran utilidad. Tal es el caso de las pizarras digitales interactivas (PDI), las que se conciben como los nuevos estilos de enseñanza-aprendizaje, lo que a su vez exige a los ejecutores de la pedagogía conocerlas para poder responder a las demandas educativas modernas. Son dos elementos que guardan una relación biunívoca, es decir el desarrollo de uno incide directamente en el otro. (Tosuntaş, Karadağ y Orhan, 2015); (Kilic, et. al. 2015).

Aunque la aparición de las pizarras digitales interactivas (PDI) parezca aún algo novedoso, la realidad es que esta tecnología fue desarrollada a principios de los noventa por Smart Technologies y comenzó a ser utilizada en las escuelas inglesas en esa misma década (Higgins, Beauchamp y Miller, 2007). A lo largo del tiempo este dispositivo ha recibido diferentes nombres, conociéndose como pizarra interactiva, pizarra electrónica o pizarra táctil. En términos técnicos la PDI es descrita como "una pantalla sensible al tacto que funciona en conjunto con un ordenador y un proyector (Álvarez y Martinell, 2016), aunque hoy día ya es posible conectarla con dispositivos más pequeños como los Tablet e incluso teléfonos celulares. Este artilugio tecnológico viene considerándose como una tecnología útil que mejora la motivación y el aprendizaje de los estudiantes, al tiempo que facilita la enseñanza a los docentes (Mata, et al., 2016). 
La inclusión de pizarras digitales interactivas en el proceso de enseñanza, impone cambios en las funciones cognitivas, en los objetivos del aprendizaje, supone modificar radicalmente las estrategias de enseñanza, privilegiando una participación más activa del alumno. Estos cambios es lo que se denomina la "enseñanza centrada en el alumno" o "aprender a aprender". En palabras de Lagunes et al., (2015) "Por su parte, los profesores también tendrán que procurar por si mismos la forma de estar en constante capacitación, no sólo con todo aquello que se relacione con la tecnología en general, sino también, de manera específica, con respecto a los medios que realmente necesite, ya que en la actualidad la tecnología abarca cada vez más disciplinas y los profesores no pueden quedarse con los conocimientos ya adquiridos anteriormente".

Según Baelo y Cantón, (2009) citado por Briede, et al., (2015), la revolución tecnológica ha irrumpido en los ámbitos del proceso de enseñanza aprendizaje, generando herramientas y recursos tecnológicos que viabilizan estrategias didácticas, apoyadas en estos medios para facilitar la construcción de conocimientos. No obstante, el uso exitoso de estas pizarras se debe a la integración adecuada de la tecnología al plan de estudio con el objetivo de desarrollar un aprendizaje personalizado y colaborativo. Por tal motivo el profesor ubicado en un entorno globalizador actual, tiene la exigencia de preparar a los estudiantes no solo a aprender contenidos, debe asimilarlos de forma independiente, es decir, buscar, evaluar críticamente, seleccionar la información relevante y saber utilizarla para realizar tareas o solucionar problemas (Obaco, et al., 2016). A nivel mundial una masiva introducción de las pizarras digitales interactivas se ha producido en varios países, tanto a nivel de escuelas como de universidades, convirtiéndose estas en un recurso innovador que dinamiza el proceso de enseñanza aprendizaje, permitiendo que los estudiantes puedan acceder a recursos atractivos, dominando el uso de la tecnología en el proceso de aprendizaje (Karsenti, 2016).

A pesar de que existían iniciativas aisladas desde la década de 1970, las políticas públicas de TIC en los países de América Latina y el Caribe, no se iniciaron hasta mediados de la década de 1990. Como toda política pública, su formulación y aplicación están ligadas a los contextos políticos y económicos en los cuales están inmersos los países (Guerra y Jordán, 2010). En América Latina pocos son los países que han incorporado de manera permanente el uso de este dispositivo tecnológico en las aulas (Víquez, 2014). Sin embargo, para que las pizarras digitales interactivas o inteligentes tengan un mayor impacto positivo en la enseñanza, se requiere de una cultura que esté abierta a los cambios en las formas de aprendizaje, y que la planificación estratégica de la formación de los futuros educadores se oriente a una didáctica del siglo XXI (Šumak et al., 2017). El Ecuador es un país en vías de desarrollo donde todavía no se cuenta en su totalidad con equipos de tal naturaleza en todas las instituciones, tomando en cuenta que dentro de las políticas de estado consta en el artículo 347 numeral 8 de la Ley Orgánica de Educación Intercultural (LOEI), el reconocimiento como responsabilidad del estado: "Incorporar las tecnologías de la información y comunicación en el proceso educativo y propiciar el enlace de la enseñanza con las actividades productivas o sociales (Obaco et al., 2016).

El uso de pizarras inteligentes proporciona una enseñanza más dirigida por el docente, le permite obtener liderazgo desde una perspectiva de tiempo, control y evaluación formativa. También, permite motivar al alumno e ir aprendiendo con él y aplicar varias formas de enseñanza al mismo tiempo, aumentando las posibilidades de aprendizaje de los alumnos (De Vita et al., 2014). El factor principal que podría facilitar el buen uso de las PDI, es que los diseños pedagógicos de los profesores, los cuales son cruciales para el aprendizaje, se adecuen y desarrollen en función de las pizarras digitales interactivas (Jang y Tsai, 2012). Estas tecnologías permiten que los procesos cognitivos ocupen un papel más significativo en el aprendizaje. Es un esfuerzo que será recompensado debido a que la práctica pedagógica, utilizando pizarras digitales interactivas como recurso didáctico, favorece el proceso de enseñanza, el desarrollo de nuevas alternativas de aprendizaje porque potencia la interacción entre el profesor y los estudiantes. La PDI ha llegado para quedarse (Carneiro et al., 2009).

Desde el punto de vista educativo, la pizarra digital interactiva presenta numerosas ventajas: a) es un recurso muy flexible, capaz de adaptarse a metodologías muy distintas, desde las más tradicionales a las más innovadoras, y a distintos estilos de enseñanza y aprendizaje; b) como heredera de la pizarra tradicional, es relativamente fácil de manejar, lo cual garantiza que incluso los docentes más pasivos ante las innovaciones tecnológicas aprendan a utilizarla con poco esfuerzo. Tal capacidad fomenta el interés tecnológico de los profesores, disminuye el estrés ante las innovadoras TIC, refuerza su autoestima y capacitación profesional y favorece la innovación pedagógica; c) si el ordenador al que está conectada dispone a su vez de conexión a Internet, la pizarra pone a disposición de alumnos y profesores la mayor biblioteca de recursos educativos concebible: la Red; d) permite proyectar sobre la pantalla la información procedente de los periféricos conectados al ordenador (entre otros, cámaras digitales de vídeo, lectores o visores de documentos, escáneres, fuentes de sonido digitales, etc.), lo cual amplía considerablemente las posibilidades de uso, pues cualquier entorno o ámbito de la realidad puede utilizarse como material didáctico; e) la conexión a Internet y el sistema de proyección hacen posible que las comunicaciones a distancia en las que intervienen audio y vídeo (por ejemplo, las videoconferencias), sean utilizadas como recurso didáctico en el aula. 
Esta posibilidad permite "romper" las fronteras de las aulas y facilita la apertura de estas a entornos de enseñanza-aprendizaje muy diversos; f) favorece el aprovechamiento de muchos materiales ya existentes, tanto realizados por los profesores como creados por terceros, como las administraciones educativas y las editoriales: presentaciones, documentos estáticos, páginas web, gráficos, fotografías, ilustraciones, carteles y posters, animaciones, líneas de tiempo, esquemas y mapas conceptuales, simulaciones, vídeos, películas, etc. En muchos casos, los docentes pueden proyectar sobre la pizarra materiales de una calidad y complejidad tales que serían imposibles de utilizar con medios convencionales; g) hace posible la reutilización del esfuerzo docente, además, dado que diversos modelos y sistemas contemplan la posibilidad de grabar una clase o acción didáctica y reproducirla de nuevo, permiten la reutilización y el análisis de las improvisaciones que son parte esencial del trabajo docente; h) permite los aprendizajes activos de los alumnos, dado que estos pueden utilizarla en todo tipo de tareas: exposiciones, realización de ejercicios interactivos, presentación y evaluación de sus trabajos, celebración de debates, etc. Este aspecto supone un refuerzo muy positivo de la motivación de los alumnos; i) en muchos casos, representa un ahorro de costes con respecto a las aulas de Informática tradicionales, en las que es necesario un ordenador para cada alumno, o para cada dos (Zhang, He, y Zheng, 2012). (Jenkins, 2016). Sin embargo, se destaca en la literatura científica algunas desventajas 0 inconvenientes respecto al uso de las PDI en el proceso de enseñanza aprendizaje. En tal caso Coscollola (2011), Unser (2017), refieren que su uso provoca que el profesor dedique más tiempo a la preparación de las actividades docentes, a su formación didáctica tecnológica, sin soslayar que se deben minimizar problemas de conexión a Internet, así como el mantenimiento correcto a los equipos.

\section{Uso de las PDI en la Universidad de Otavalo}

Todo centro educativo que trata de mostrar el nivel tecnológico de sus enseñanzas, que tiende a estar en la cima de la calidad educativa, debe considerar la conveniencia del uso de las PDI. La Universidad de Otavalo, es la única institución ubicada en la zona norte del país, que tiene instaladas las PDI en todas sus aulas, por lo que no es posible la comparación con otras universidades del Ecuador. La PDI ofrece al docente, acostumbrado a las pizarras tradicionales de tiza o de rotuladores, encontrar un recurso muy cercano a la tradición pedagógica que incorpora las TIC en el aula de manera visible y transparente. Todos los alumnos pueden ver y actuar con los equipos informáticos, individual o grupalmente ante todos sus compañeros. Es necesario aceptarla progresivamente como un recurso ineludible para las demandas pedagógicas existentes, de forma tal que se trasformen los ambientes educativos en consonancia con los requerimientos conceptuales del siglo XXI. Esto no significa reemplazar al docente como guía del proceso de enseñanza aprendizaje, sino que demanda de éstos nuevos enfoques para la clase y nuevas habilidades, llegando a suponer un verdadero desafío.

La PDI hace posible una amplia variedad de especificaciones y capacidades, por ejemplo: 1) la manipulación fácil y rápida de textos e imágenes; 2) tomar apuntes digitales; 3 ) utilizar la Web y sus recursos ante toda la clase; 4) mostrar videos y facilitar el debate; 5) utilizar y demostrar diferentes tipos de software; 6) guardar notas para la posterior revisión; 7) utilizar el e-mail para proyectos colaborativos inter centros; 8) crear lecciones digitales con imágenes y sonidos; 9) escribir y resaltar los aspectos de interés sobre textos, imágenes o vídeos; 10) utilizar todas las técnicas y recursos de presentación; 11) facilitar la presentación de trabajos de los alumnos. Por tanto, la innovación toca a las universidades y otras instituciones de educación superior, por lo que debe planificarse estrategias que estimulen el autoaprendizaje, así como la actualización de los profesionales y personal de apoyo a la docencia, que sirvan de sostén a la formación académica. La educación superior actual debe ser una puerta de acceso a la sociedad del conocimiento, quizá la puerta más importante por su situación privilegiada para la generación y transmisión del saber humano. En la sociedad del conocimiento, la universidad tradicional coexistirá con universidades virtuales y con otras formas de universidad. Por tal motivo, la Universidad de Otavalo, en su continuo perfeccionamiento por alcanzar la excelencia en las funciones sustantivas de la educación superior, se trazó desde el año 2009 como meta impostergable pasar del patrón de universidad económicamente viable, a aquella que juzga la verdadera necesidad, no sólo desde el punto de vista de la formación profesional sino, también, de su contribución a la construcción de una nueva sociedad. Por ello incorporó a su proceso de enseñanza aprendizaje las PID, en todas sus aulas, convirtiéndose en pionera en el uso de este recurso didáctico en la zona norte del Ecuador.

\section{MATERIALES Y MÉTODOS}

Para corroborar los efectos del uso de las PDI en el proceso de enseñanza aprendizaje, en la Universidad de Otavalo se realizó un estudio preliminar de corte exploratorio a docentes y estudiantes, utilizando el enfoque cuantitativo, es decir aplicándole un único instrumento con ítems comunes a todo el personal docente a tiempo completo (25 docentes) y a una muestra estratificada de estudiantes de la institución, (206) con un nivel de confianza del 95\%. Se usó el paquete estadístico SPSS versión 21 para el procesamiento de la encuesta con el objetivo de alcanzar de forma expedita los resultados, representando las respuestas en diferentes tablas. El uso del enfoque cuantitativo, permite que el resultado de la investigación esté bien 
delimitado y contextualizado en el entorno en que se está analizando el problema, lo que posibilita argumentar con bases sólidas los resultados alcanzados de forma descriptiva. Se usó la escala Lickert, lográndose su uso homogéneo en todas las preguntas de la encuesta, donde las respuestas fueron presentadas con tres niveles de medición, Mucho, Poco y Nada, siendo el Mucho una valoración positiva, el Poco una valoración intermedia y el Nada una valoración negativa. Por ser una investigación de tipo exploratoria se consideró necesario acotar a tres niveles de respuesta la escala Lickert, en aras de obtener respuestas más concretas. La estructura del cuestionario tanto para docentes como para estudiantes se inicia con un encabezado e instrucciones. Abarca en total 7 aspectos, 3 preguntas de carácter informativo (género, edad y semestre académico que cursa). Posteriormente se distribuyen 18 preguntas en cuatro categorías: Categoría 1: Nivel de conocimientos, con 4 preguntas en la escala de excelente, muy bueno, bueno y regular. Categoría 2: Ventajas del uso de la PDI. Categoría 3: Desventajas del uso de la IDS. Estas 2 categorías tienen 4 preguntas cada una con la escala Lickert. Categoría 4: Incidencia del uso de la PDI con 6 preguntas; las 3 primeras con la escala de siempre, a veces, casi nunca y nunca y las 3 restantes con la escala Lickert. Una vez elaborado el cuestionario se requirió la valoración de 5 expertos en las áreas de TIC, profesores y tecnicos de nivel superior del departamento de Sistemas y también de un profesor experto en el área pedagógica de la Universidad de Otavalo, los que observaron que los ítems propuestos responden al logro de los objetivos establecidos. Sin embargo se hicieron sugerencias acerca de cómo presentar la diagramación de las escalas propuestas y su tipo de encabezado. Los primeros 7 aspectos de la encuesta fueron respondidos por estudiantes y profesores de la muestra, mientras que las 2 últimas preguntas estuvieron dirigidas a los docentes de la muestra. Las posibles respuestas se clasificaron como mucho, poco y nada.

\section{RESULTADOS Y DISCUSIÓN}

Luego del procesamiento de las encuestas se obtuvo la información siguiente: 1) el uso de las PDI ayuda al alumno a entender mejor el contenido de los temas; 2) su uso de la PDI aumenta la participacion del alumno en clases; 3) el empleo de la PDI hace las clases mas interesantes e interactivas; 4) aumenta la atencion del alumno; 5) el profesor dispone de herramientas que facilitan el proceso de enseñanza; 6) la PDI aumenta las oportunidades de participación y discusión en las clases; 7) se trata de una tecnologia sencilla de utilizar; 8) para el docente es mas laborioso planificar una clase con la PDI; Y 9) el uso de la pantalla interactiva facilita una renovación metodológica que promueve la inovación didáctica.

En la tabla 1 se muestran los resultados obtenidos para los 7 aspectos comunes a los 231 encuestados tanto docentes como estudiantes. Como se puede observar en la tabla 1 para las 7 preguntas se obtuvo un resultado favorable, siendo la respuesta Mucho la que ocupó el mayor porcentaje. En la figura 1 se muestra el resultado para la pregunta 5, sobre la optimización del tiempo en el proceso de enseñanza por parte del profesor. En la tabla 2 se comparan los criterios de los docentes y estudiantes que respondieron positivamente a las preguntas comunes de la encuesta. En la tabla 2 se muestra, la pregunta sobre cómo ayuda a entender a los alumnos el contenido de los temas de estudio, se manifiesta una sobre estimación leve por parte de los docentes, con un $72 \%$ sobre el $66 \%$ de los estudiantes que lo valoran positivamente. Algo similar pero en mayor medida se revela para la segunda pregunta de la encuesta, que se refiere a la participación de los estudiantes en las clases, donde los docentes sobre valoran el efecto de las pizarras interactivas en un $18 \%$ más que los estudiantes.

Tabla 1: Resultado de las preguntas comunes a docentes y estudiantes

\begin{tabular}{|c|c|c|c|}
\hline \multirow{2}{*}{ Preguntas } & \multicolumn{3}{|c|}{$\begin{array}{l}\text { Resultados de la } \\
\text { Encuesta }\end{array}$} \\
\hline & Mucho & Poco & Nada \\
\hline $\begin{array}{l}\text { 1. El uso de las pantallas digitales interactivas ayuda al alumno e entender mejor el } \\
\text { contenido de los temas }\end{array}$ & $67,1 \%$ & $30,7 \%$ & $2,2 \%$ \\
\hline $\begin{array}{l}\text { 2. El uso de la pantalla digital interanctiva aumenta la participacion del alumno en } \\
\text { clases }\end{array}$ & $56,7 \%$ & $38,1 \%$ & $5,2 \%$ \\
\hline 3. El uso de las PDI hace las clases mas interesantes e interactivas a los alumnos & $66,2 \%$ & $31,2 \%$ & $2,6 \%$ \\
\hline $\begin{array}{l}\text { 4. Mediante la presentacion de trabajos atractivos en la PDI aumenta la atencion del } \\
\text { alumno }\end{array}$ & $61,9 \%$ & $36,4 \%$ & $1,7 \%$ \\
\hline 5. El uso de la PDI optimiza el tiempo que el docente dispone para enseñar & $58,9 \%$ & $36,8 \%$ & $4,3 \%$ \\
\hline 6. La PDI aumenta las oportunidades de participacion y discusión en las clases & $56,3 \%$ & $40,2 \%$ & $3,5 \%$ \\
\hline 7. Se trata de una tecnologia sencilla de utilizar & $68,0 \%$ & $29,0 \%$ & $3,0 \%$ \\
\hline
\end{tabular}




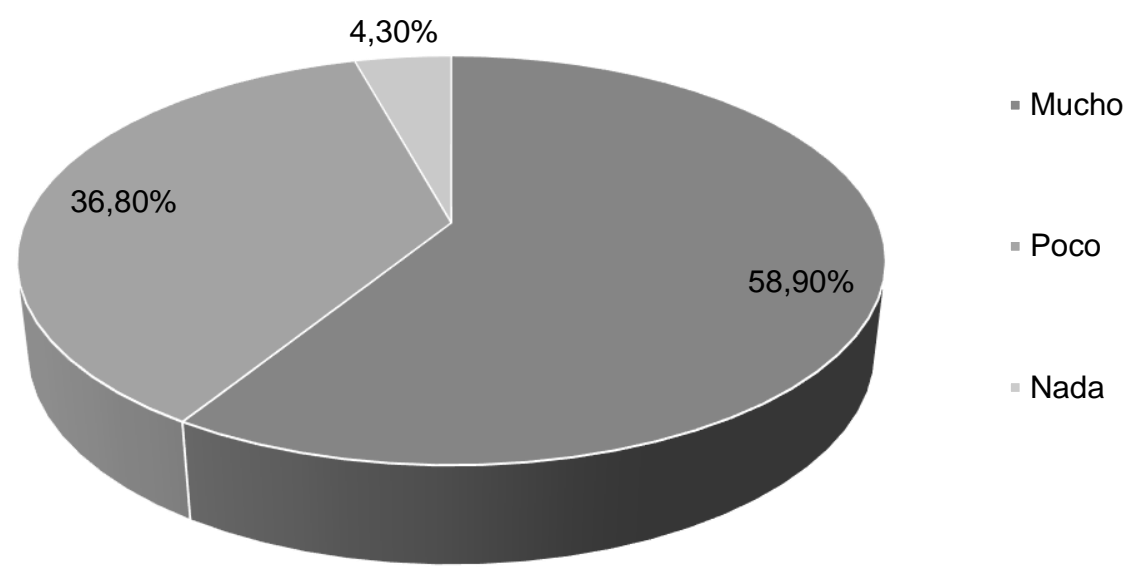

Fig. 1: El uso de la pantalla interactiva optima el tiempo que el docente dispone para enseñar

Para analizar los índices de aceptación por parte de los estudiantes y docentes al uso de las pizarras interactivas digitales en la Universidad de Otavalo, se construyó la tabla 2 para cada una de las preguntas analizando solamente las repuestas positivas Mucho y analizando los índices de aceptación entre estudiantes y docentes.

Tabla 2: Resultados para la respuesta Mucho

\begin{tabular}{|c|c|c|c|}
\hline \multirow{2}{*}{ Pregunta } & \multicolumn{3}{|c|}{ Mucho } \\
\hline & $\%$ total & $\%$ docentes & $\%$ alumnos \\
\hline $\begin{array}{l}\text { 1. El uso de las pantallas interactivas ayuda al alumno } \\
\text { e entender mejor el contenido de los temas }\end{array}$ & 67,0 & 72,0 & 66,4 \\
\hline $\begin{array}{l}\text { 2. El uso de la pantalla interanctiva aumenta la } \\
\text { participacion del alumno en clases }\end{array}$ & 56,7 & 72,0 & 54,8 \\
\hline $\begin{array}{l}\text { 3. El uso de la pantalla interactiva hace las clases mas } \\
\text { interesantes e interactivas a los alumnos }\end{array}$ & 66,2 & 64,0 & 66,5 \\
\hline $\begin{array}{l}\text { 4. Mediante la presentacion de trabajos atractivos en la } \\
\text { pizarra interactiva aumenta la atencion del alumno }\end{array}$ & 61,9 & 48,0 & 63,6 \\
\hline $\begin{array}{l}\text { 5. El uso de la pantalla interactiva optimiza el tiempo } \\
\text { que el docente dispone para enseñar }\end{array}$ & 58,9 & 64,0 & 58,3 \\
\hline $\begin{array}{l}\text { 6. La pantalla interactiva aumenta las oportunidades de } \\
\text { participacion y discucion en las clases }\end{array}$ & 56,3 & 68,0 & 54,9 \\
\hline 7. Se trata de una tecnologia sencilla de utilizar & 68,0 & 68,0 & 68,0 \\
\hline
\end{tabular}

En la pregunta 4 se nota una mayor valoración por parte de los estudiantes al efecto de presentación de trabajos atractivos en la pizarra y cómo aumenta su atención en clase, lo que resulta muy revelador pues precisamente esto implica la necesidad de un cambio metodológico por parte de los docentes al hacer uso de las pantallas interactivas. Es de destacar que en trabajos publicados por otros autores se encontraron resultados similares, donde los estudiantes disfrutaron llegar a la pizarra y estaban ansiosos por participar (Olsen et al., 2011); (Lan y Hsiao, 2011); (Shenton y Pagett, 2007)

La utilización de las PDI como elemento innovador del proceso de enseñanza aprendizaje, tiene ventajas para los estudiantes. Su utilización despierta el interés en ellos para el uso de nuevas tecnologías, lo que provoca que durante la clase estén más motivados, al ser un recurso atractivo y al poder acceder a gran cantidad de recursos educativos y entornos de aprendizaje. Esto hace que las explicaciones sean más amenas, y el estudiante aprenda en menos tiempo. Se debe prestar atención a la baja valoración que los docentes de la Universidad de Otavalo le dan a este punto, solo un $48 \%$, lo que refleja un bajo interés en perfeccionar la metodología tradicional, en aras de una presentación motivante más adecuada con el nuevo entorno interactivo existente. 
En la pregunta 6 que trata sobre si el uso de la pantalla interactiva aumenta las oportunidades de participacion y discusión en las clases se vuelve a encontrar una leve sobre valoración por parte de los docentes en relación a los estudiantes, que aunque lo consideran un hecho positivo lo evalúan solo en un $55 \%$, lo cual esta de acuerdo con los resultados obtenidos por Jenkins, D. (2016), en su tesis doctoral. Las preguntas 3,5 y 7 recibieron aproximadamente la misma estimación por parte de estudiantes y docentes. Es significativo que las respuestas a todas las preguntas fueron positivamente mayores a un $50 \%$, para la muestra total de docentes y estudiantes, lo que manifiesta la aceptación de esta innovadora tecnología y se valoran sus aspectos positivos; sin embargo en ninguno de las respuestas se obtiene una puntuación mayor al $68 \%$ de aceptación, por lo que se hace necesario perfeccionar los métodos de enseñanza utilizados por los docentes y el logro de una mayor explotación de las pizarras interactivas por parte de los estudiantes, sobre todo en su estudio individual y grupal extra clase. Con el fin de corroborar si la diferencia de criterios entre estudiantes y docentes resultan estadísticamente significativas, en las preguntas 2, 4 y 6 se realizó un análisis de constatación de hipótesis, utilizando chi cuadrada para tres filas y dos columnas, con 2 grados de libertad. Con la utilización del SPSS 21 resultó que se cumple la hipótesis nula y no existe significancia estadística entre estudiantes y docentes.

Esta problemática de lograr una mayor explotación de las PDI se puede encontrar en general al analizar el uso de las TIC por parte de los docentes universitarios. Se hace necesario la impartición de cursos de actualización de conocimientos tecnológicos y pedagógicos en el profesorado universitario, como un elemento clave en el proceso de formación continua que debe ser promovido por las universidades (LagunesDomínguez, et al., 2015; Morales et al., 2015). Una solución a este problema según Jenkins, (2016), es ofrecer a los docentes capacitación continua en forma de videos instructivos en línea. Si los videos están bien organizados, son consistentes, se crean en alta calidad y la instrucción es un andamio para apoyar el aprendizaje del profesor, se pueden usar para ayudar a los educadores a implementar herramientas más atractivas en su lección y ayudar al aprendizaje del alumno. Con este apoyo, los maestros tendrán el conocimiento que necesitan para desarrollar lecciones PDI para ayudar a apoyar el aprendizaje de los estudiantes en la era digital. En la tabla 3 se muestra el resultado de las preguntas 8 y 9 de la encuesta, las cuales fueron formuladas solo a los docentes.

Tabla 3: Respuesta a preguntas solo para docentes.

\begin{tabular}{|l|l|l|l|}
\hline \multicolumn{1}{|c|}{ Pregunta } & Mucho & Poco & Nada \\
\hline $\begin{array}{l}\text { Para el docente es mas laborioso planificar una clase para la } \\
\text { pantalla interactiva }\end{array}$ & $32 \%$ & $68 \%$ \\
\hline $\begin{array}{l}\text { El uso de la pantalla interactiva facilita una renovación } \\
\text { metodológica que promueve la inovacion didáctica }\end{array}$ & $76 \%$ & $24 \%$ & \\
\hline
\end{tabular}

De la tabla 3 se concluye que los docentes consideran poco o nada laborioso la planificación de las clases utilizando las pantallas interactivas, lo que denota su fácil uso y aplicación al proceso docente educativo, Sin embargo, esto puede confundirse con la fácil adaptación de las formas tradicionales de enseñanza al entorno tecnológico de las pizarras interactivas, lo que resulta poco adecuado y para nada innovador en el desarrollo y utilización de nuevas metodologías, acordes con el mayor número de posibilidades que brinda la pantalla digital interactiva. Relacionado con este aspecto Jenkins, D (2016) considera que las pizarras interactivas tienen el potencial de revolucionar la forma en que la información es presentada al estudiante del siglo veintiuno. Son un conglomerado de tecnologías de enseñanza previas, como pizarra, sistema de audio, Internet, etc. Desafortunadamente, la falta de capacitación de los docentes en estos dispositivos deja mucho que desear cuando se trata de las lecciones que imparten.

En este aspecto los resultados obtenidos por Tapasco y Giraldo (2017) en México arrojaron que con relación a los cuestionamientos sobre la percepción que los profesores tienen sobre las TIC, el $65.3 \%$ de los docentes encuestados consideran que preparar un curso con apoyo de la TIC, es más exigente que preparar un curso tradicional, esta diferencia de criterios puede estar relacionada con la mayor o menor exigencia metodológica a la hora de utilizar e integrar las PDI en la enseñanza universitaria. La respuesta por parte de los docentes a la última pregunta de la encuesta es alentador, ya que supone una aceptación del $76 \%$ de los encuestados a que el uso de la pantalla interactiva facilita una renovación metodológica, que promueve la inovacion didáctica, siendo este el punto más álgido para su perfeccionamiento en las clases.

A partir de los resultados obtenidos en la encuesta se propone que dentro de la semana académica que se ejecuta al final de cada semestre, se realice un taller para analizar las experiencias por facultades en el uso de las PDI, para debatir y promover eficientemente su aplicación, donde participen docentes y estudiantes 
invitados con el objetivo de obtener información histórica para el desarrollo de estudios longitudinales sobre el uso de las PDI y su integración en las aulas universitarias de Otavalo, resaltando sus resultados y contribución a la mejora del aprendizaje. Por tanto. la utilización de la pantalla interactiva en la Universidad de Otavalo ya cumple 8 años como un auxiliar inapreciable en el proceso enseñanza aprendizaje, muchos son los aciertos y deficiencias que aun persisten en el camino del perfeccionamiento metodológico necesario para su mejor utilización y aumento de su eficiencia

\section{CONCLUSIONES}

Tanto docentes como estudiantes ven positivamente el uso de las pizarras interactivas en las aulas universitarias, lo que ayuda al alumno a entender mejor el contenido de los temas, hace las clases más interesantes e interactivas, es una tecnología de facil aplicación que estimula la participación del estudiante en el proceso educativo.

Solo el $48 \%$ de los docentes estiman que mediante la presentación de trabajos atractivos en la pizarra interactiva aumenta la atención del alumno, sin embargo los estudiantes valoran este hecho como muy positivo para una aceptación del $64 \%$. Ello puede estar relacionado con la falta de un planificado y sistemático proceso de perfeccionamiento docente, para explotar en mayor medida la integración de las pizarras interactivas al proceso de enseñanza aprendizaje en la educación superior, lo que obligatoriamente pasa por el desarrollo de nuevas metodologías y/o estrategias para la mejora de las ya existentes, que hagan más atractivas las clases a los estudiantes y le faciliten el acceso a la información en línea, apoyándose en el vertiginoso desarrollo de la TIC; sobre todo cuando los organismos de control de Ecuador están enfrascados en la supervisión y aprobación de los rediseños de carreras que tributan al perfeccionamiento de la educación superior en el país, por lo que se hace imprescindible tener en cuenta la integración armónica del plan de estudio, su desagregación en los sílabos, la planificación del auto aprendizaje del estudiante, la capacitación del docente en función del uso de este recurso tecnológico, entre otras cuestiones.

Es necesario acotar que por la naturaleza del estudio que es básicamente exploratorio, las conclusiones son limitadas, pero por ser parte de un proyecto de investigación se harán otras valoraciones teniendo en cuenta experiencias internacionales de países del área.

\section{REFERENCIAS}

Álvarez, R.A. y A.R. Martinell, La pizarra digital: recurso didáctico para la visualización e interacción académica en educación superior, ISSN: 1659-0104, Ensayos Pedagógicos, 10(2), 149-166 (2016)

Basantes, A.V., M. E. Naranjo, M. C. Gallegos y N. M. Benítez, Los Dispositivos Móviles en el Proceso de Aprendizaje de la Facultad de Educación Ciencia y Tecnología de la Universidad Técnica del Norte de Ecuador, doi: 10.4067/S071850062017000200009, Formación Universitaria, 10(2), 79-88 (2017)

Briede, J., I. Leal, M. Mora y C. Pleguezuelos, Propuesta de Modelo para el Proceso de Enseñanza-Aprendizaje Colaborativo de la Observación en Diseño, utilizando la Pizarra Digital Interactiva (PDI), doi: 10.4067/S071850062015000300003, Formación Universitaria, 8(3), 15-26 (2015)

Carneiro, R., J.C. Toscano e Y. Díaz, Los desafíos de las TIC para el cambio educativo, $1^{\text {a }}$ Ed., OEI - Fundación Santillana, Madrid, España (2009)

Coscollola, M., Pizarra Digital Interactiva en el aula: Uso y valoraciones sobre el aprendizaje, Estudios sobre educación, Universidad de Navarra (2011)

De la Herran, A. e I. Fortunato, La clave de la educación no está en las nuevas Tecnologías de la Información y la Comunicación (TIC), doi: 10.4025/actascieduc.v39i3.33008, Acta Scientiarum, Education, 39(3), 311-317 (2017)

De Vita, M., L. Verschaffel y J. Elen, Interactive whiteboards in mathematics teaching: A literature review, doi: 10.1155/2014/401315, Education Research International, 1-16 (2014)

Gallego, D. J., M. L. Cacheiro y J. Dulac, La pizarra digital interactiva como recurso docente. Teoría de la Educación, Educación y Cultura en la Sociedad de la Información, Revista Electrónica Teoría de la Educación, Educación y Cultura en la Sociedad de la Información, ISSN: 1138-9737, 10(2), 127-145 (2009)

Guerra, M. y V. Jordán, Políticas públicas de la sociedad de la información en América Latina: ¿una misma visión?, Naciones Unidas, Santiago de Chile (2010)

Gómez, M. G. y L. G. Aretio, La formación como factor clave en la integración de la Pizarra Digital Interactiva. Perspectivas de profesores y coordinadores TIC, doi: 10.6018/reifop.19.3.225451, Revista Electrónica Interuniversitaria de Formación del Profesorado, 19(3), 35-51 (2016)

Hernández, M.R., V. M. Rodríguez, F.J. Parra y P. Velázquez, Las Tecnologías de la Información y la Comunicación (TICs) en la Enseñanza-Aprendizaje de la Química Orgánica a través de Imágenes, Juegos y Videos, doi: 10.4067/S071850062014000100005, Formación Universitaria, 7(1), 31-40 (2014) 
Higgins, S., G. Beauchamp y D. Miller, Reviewing the literature on interactive whiteboards, Learning, Media and technology, 32(3), 213-225 (2007)

Jang, S.-J. y M.- F.Tsai, Reasons for using or not using interactive whiteboards: Perspectives of Taiwanese elementary mathematics and science teachers, doi: 10.14742/ajet.781, Australasian Journal of Educational Technology, 28(8), 14511465 (2012)

Jenkins, D., Understanding how Interactive Whiteboard Technology Supports Teaching and Learning, Tesis de graduación en Master of Arts in Education, California State University San Marcos, California - United States (2016)

Karsenti, T., The Interactive Whiteboard: Uses, Benefits, and Challenges. A Survey of 11,683 Students and 1,131 Teachers, doi: 10.21432/T2WW4J, Canadian Journal of Learning and Technology, 42(5) (2016)

Kilic, E., Ç. Güler, H.E. Çelik y C. Tatli, Learning with interactive whiteboards: Determining the factors on promoting interactive whiteboards to students by Technology Acceptance Model, doi: /abs/10.1108/ITSE-05-2015-0011, Interactive Technology and Smart Education,12(4), 285-297 (2015)

Lagunes-Domínguez, A., C. A. Torres-Gastelú, M. A. Flores-García y A. Rodríguez-Figueroa, Comparativo del uso de Tecnologías de la Información y Comunicación (TIC) por Profesores de dos Universidades Públicas de México, doi: 10.4067/S0718-50062015000200003, Formación Universitaria, 8(2), 11-18 (2015)

Lan, T. y T. Hsiao, A Study of Elementary School Students' Viewpoints on Interactive Whiteboard, doi: 10.3844/ajassp.2011.172.176, American Journal of Applied Sciences, 8(2), 172-176 (2011)

Mata, L., G. Lazar e I. Lazar, Effects of study levels on students' attitudes towards interactive whiteboards in higher education, doi: 10.1016/j.chb.2015.07.044, Computers in Human Behavior, 54, 278-289 (2016)

Morales Capilla, M., J. M. Trujillo Torres y F. Raso Sánchez, Percepciones acerca de la integración de las TIC en el proceso de enseñanza-aprendizaje de la Universidad, doi: 10.12795/pixelbit.2015.i46.07, Revista de Medios y Educación, 46, 103-117 (2015)

Murano, J., Pizarra digital. Herramienta metodologica integral en el contexto del aula del sigol XX, 1ㄹ Ed., 50-75, Ideaspropias, Galicia, España (2012)

Obaco, J. S. C., N. I. P. Valladares y J. P. S. Orellana, Uso de pizarras digitales interactivas como recurso de enseñanza para los docentes, doi: 10.1016/j.magis.2016.11.001, Magister, 28(2), 71-85 (2016)

Olsen, A., S. Lemire y M. Baker, The Impact of Self-Efficacy and Peer Support on Student Participation with Interactive White Boards in Middle School Mathematics Class, Journal of Computers and Science Teaching, 30(2), 163-178 (2011)

Shenton, A. y L. Pagett, From 'Bored' to Screen: the use of the interactive whiteboard for literacy in six primary classrooms in England, doi: 10.1111/j.1467-9345.2007.00475.x, Literacy, 41(3), 129-136, Steussy, L. (2012) (2007)

Stroud, R., B. Drayton, K. Hobbs y J. Falk, Interactive whiteboard use in high-tech science classrooms: Patterns of integration, International Journal of Emerging Technologies in Learning, 9(9), 41-49 (2014)

Šumak, B., M. Pušnik, M. Heričko y A. Šorgo, Differences between prospective, existing, and former users of interactive whiteboards on external factors affecting their adoption, usage and abandonment, doi: 10.1016/j.chb.2016.09.006, Computers in Human Behavior, 72, 733-756 (2017)

Tapasco, O. A. y J. A. Giraldo, Estudio Comparativo sobre Percepción y uso de las TIC entre Profesores de Universidades Públicas y Privadas; doi: 10.4067/S0718-50062017000200002, Formación Universitaria, 10(2), 03-12 (2017)

Tosuntaş, Ş.B., E. Karadağ y S. Orhan, The factors affecting acceptance and use of interactive whiteboard within the scope of FATIH project: A structural equation model based on the Unified Theory of acceptance and use of technology, doi: doi.org/10.1016/j.compedu.2014.10.009, Computers y Education, 81, 169-178 (2015)

Unser, C.E., A Study on The Positives and Negatives of Using Technology In The Classroom, Long Island University (2017)

Víquez, I., Las TIC en la educación inclusiva e influencia en el aprendizaje de preescolares, Congreso lberoamericano de Ciencia, Tecnología, Innovación y Educación 1-15, Buenos Aires, Argentina, 12, 13 y 16 de noviembre (2014)

Yang, H., J. Yu, H. Zo y M. Choi, User acceptance of wearable devices: An extended perspective of perceived value, doi: doi.org/10.1016/j.tele.2015.08.007, Telematics and Informatics, 33(2), 256-269 (2016)

Zhang, S., W. He, Q. Yu y X. Zheng, Low-cost interactive whiteboard using the Kinect. In Image Analysis and Signal Processing (IASP), 2012; doi: 10.1109/IASP.2012.6424988, IEEE Xplore, (12) 1-15 (2013) 
